\title{
Higher Čech Theory
}

\author{
TIBOR BEKE \\ ${ }^{1}$ Department of Mathematics, University of Michigan, East Hall, 525 East University \\ Avenue, Ann Arbor, MI 48109-1109, USA. \\ e-mail: tbeke@umich.edu
}

(Received: June 2004)

\begin{abstract}
We introduce a notion of 'cover of level $n$ ' for a topological space, or more generally any Grothendieck site, with the key property that simplicial homotopy classes computed along the filtered diagram of $n$-covers biject with global homotopy classes when the target is an $n$-type. When the target is an Eilenberg-MacLane sheaf, this specializes to computing derived functor cohomology, up to degree $n$, via simplicial homotopy classes taken along $n$-covers. Our approach is purely simplicial and combinatorial.
\end{abstract}

Mathematics Subject Classifications (2000): 18G55, 55N30

Key words: C̆ech Technology, Grothendieck topos, groupoids

\section{Introduction}

The well-known theory of Čech cohomology with coefficients in a (pre) sheaf has been extended by Grothendieck and his collaborators from topological spaces to arbitrary Grothendieck topologies. Čech cohomology of sites is isomorphic to their derived functor cohomology, in general, only in dimensions 0 and 1 . Verdier has introduced a variant of the notion of cover in such a way that the cohomology groups computed along his 'hypercovers' are isomorphic to derived functor cohomology in all dimensions, for arbitrary sites. The starting point for this research was the following naïve but (perhaps) irresistible question: Is there a notion of 'cover of level $n$ ' with respect to which Čech covers have level 1, and get the cohomology right up to dimension 1 , while Verdier covers have level $\infty$, and get the cohomology right in all dimensions?

The answer is (as the author discovered after most of this research was completed) that while the term, 'truncated hypercovering of level $n$ ', does make an appearance in [2], p. 96 (see also Verdier's Appendice to SGA4, fascicule 1 , exposé 5 for the original description) its properties seem to be mostly unexplored. But there are good reasons for such an exploration. One is liberating $n$-covers from the formalism of sites (which makes them look much less similar to plain simplicial sets than they really are) and the other is to embed them in a theory of Postnikov sections, which makes the 
formalism applicable to coefficients more general than Eilenberg-MacLane spaces (i.e. abelian ones).

Recall that the data for an open cover of a topological space can be assembled into a simplicial sheaf with distinguished properties, and hypercovers are simplicial sheaves by definition. The notion of $n$-cover is cast in a simplicial language, and is defined intrinsically in terms of the topos, i.e. the category of sheaves on the site. We also introduce a notion of Cech $n$-cover that is specific to a site; these are genuine combinatorial objects, standing in the same relation to $n$-covers as topological open covers do to the simplicial sheaf they give rise to.

One of the several equivalent definitions of abelian sheaf cohomology is

$$
H^{n}(\mathcal{E}, A)=\text { ho }_{\mathcal{E}^{\Delta \mathrm{op}}}(\mathbf{1}, \widetilde{K(A, n)})
$$

Here $\mathcal{E}=\operatorname{Sh}(\mathcal{C}, J)$ is the category of set-valued sheaves on a site, $A$ an abelian sheaf, ho ${ }_{\mathcal{E}^{\Delta \mathrm{p}}}$ stands for the category of simplicial objects in $\mathcal{E}$ with the local weak equivalences (formally) inverted, 1 is the terminal object of $\varepsilon^{\Delta^{\text {op }}}$ and $\widetilde{K(A, n)}$ is a simplicial Eilenberg-MacLane sheaf corresponding to $A$ in degree $n$. Level $n$ Cech cohomology will be defined as a filtered colimit of simplicial homotopy classes whose source is an $n$-cover; from the viewpoint of simplicial homotopy theory, the target could be an arbitrary simplicial sheaf (so perhaps 'level $n$ Čech homotopy' would be a better name) and the main result becomes the following

THEOREM. Write $\left[\operatorname{cov}_{\mathcal{E}}^{n}\right]$ for the category with objects the $n$-covers of $\mathcal{E}$ and morphisms simplicial homotopy classes of maps. [ $\left.\operatorname{cov}_{\mathcal{E}}^{n}\right]$ is a cofiltered category possessing cofinal small subcategories. Let $X$ be a simplicial object in $\mathcal{E}$. Writing $[-, X]$ for the contravariant 'simplicial homotopy classes' functor $\mathcal{E}^{\Delta^{o p}} \rightarrow$ Set, one has a natural map (of sets)

$$
\underset{\left[\operatorname{cov}_{\varepsilon}^{n}\right]}{\operatorname{colim}}[-, X] \rightarrow \mathrm{ho}_{\mathcal{E}^{\Delta p p}}(\mathbf{1}, X)
$$

which is a bijection if $X$ is an exact $n$-type.

There is an analogous statement for Čech $n$-covers. An exact $n$-type is, in turn, a particularly good simplicial model for a (local, in the case of sheaves) weak homotopy type with vanishing local homotopy groups above dimension $n$. For example, of all simplicial sets of the weak homotopy type of an Eilenberg-MacLane space $K(G, 1)$, the nerve of $G$ is also exact. Any homotopical $n$-type, taken in the ordinary sense, possesses models that are exact $n$-types. The concept, which is due to Duskin, will be discussed in detail.

As a corollary of the main theorem, the Čech cohomology groups of level $n$ "get cohomology correctly" (i.e. are isomorphic to derived functor 
cohomology) in dimensions 0 through $n$. Setting $n=1$ and $X=K(G, 1)$ (here $G$ being a sheaf of groups), one recovers Grothendieck's theorem on (not necessarily abelian) $H^{1}$ and its computability by 'level 1', that is ordinary Čech cohomology. The preservation of algebraic structure on homotopy classes - for example, in case $X$ is a group or abelian group object up to homotopy-follows by naturality.

The 0,1 and 2-covers can be economically reindexed as groupoids with extra structure, and it might be thought that $n$-covers, as defined here, could shed light on the elusive notion of ' $n$-groupoids' or 'weak $n$-categories'. That is not so, one can see, already for $n=2$. The reason is that $n$-covers are defined via horn- and simplex-filling conditions, i.e. purely existential conditions, while for groupoids and similar algebraic structures one expects operations and identities - such as functional or functorial choices of fillers.

The reader who is impatient (or turned away by the prevalence of the term 'topos') but wishes to get a feel for the subject is encouraged to look at Theorem 3.15 and Proposition 3.16-showing how cleanly one can 'chop off' Verdier covers, and the main property of Verdier covers, at a finite stage; or at the discussion on the last few pages, where we give an example of a sheaf cohomology class that is not supported on any Čech cocycle (in the classical sense) but can be represented by a higher Cech cocycle (in the sense of this paper).

The proof of the main theorem is quite straightforward; it employs no additive tools such as chain complexes or spectral sequences, but proceeds purely by local manipulations on simplicial objects, mainly with Postnikov sections. One reason for the length of this paper is the number of 'service paragraphs' it contains, somewhat independent but (it is hoped) enjoyable illustrations of the various notions used. Though the intrinsic level of generality of our results is that of simplicial sheaves on any Grothendieck site, essentially all proofs can be phrased in direct combinatorial terms involving plain simplicial sets. The very first such 'service paragraph', entitled For logical reasons, explains just how this is possible; it is a depository of some sheaf-theoretic technologies that were only perfected after the publishing of SGA4. These tricks, constantly used by a handful of 'experts', make proofs both more streamlined and more general, often eliminating assumptions of 'enough points' on sites.

\subsection{NOTATIONAL AND TERMINOLOGICAL CONVENTIONS}

A topos is a category abstractly equivalent to the category of Set-valued sheaves on some Grothendieck site. To minimize set-theoretical issues, we assume that our toposes can always be written as the category of sheaves on a small site. Under this assumption, a topos will possess a proper class 
of objects and small hom-sets in the usual sense, and there won't be a need to adjoin extra set-theoretical universes.

As general background on sites, toposes and geometric morphisms, we recommend the first three chapters of MacLane-Moerdijk [16].

Write ho $\varepsilon_{\varepsilon^{\Delta \mathrm{po}}}$ for $\varepsilon^{\Delta^{\mathrm{op}}}\left[\mathrm{W}^{-1}\right]$, the category of simplicial sheaves with the class of local weak equivalences inverted. This is a locally small category (i.e. between any two objects there is a hom-set rather than proper class of morphisms); this can proved on purely set-theoretic grounds, or follows from $W$ being part of a Quillen model category structure on $\varepsilon^{\Delta^{\text {op }}}$. We will not use any model-theoretic arguments, though.

The symbol 1 will always stand for a terminal object, in the category that's clear from the context.

\section{For Logical Reasons}

Let us illustrate the point with an example that is needed in this paper.

PROPOSITION 2.1. Let

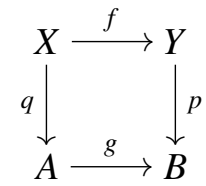

be a pullback diagram in SSet, with $p$ a Kan fibration. Assume $g$ is an $n$-equivalence, that is, it induces isomorphisms on $\pi_{i}$ for $0 \leqslant i \leqslant n$, and in addition it induces an epi on $\pi_{n+1}$ (for all basepoints). Then $f$ is an n-equivalence.

PROPOSITION 2.2. Let $\mathcal{E}$ be a topos and

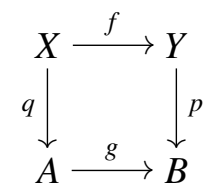

a pullback diagram in $\mathcal{E}^{\Delta^{o p}}$, with $p$ a local Kan fibration. Assume $g$ is a local $n$-equivalence, that is, it induces isomorphisms on the sheaves $\pi_{i}$ for $0 \leqslant i \leqslant n$, and in addition it induces an epi on $\pi_{n+1}$ (for all local basepoints). Then $f$ is a local n-equivalence.

Proof of 2.1. Consider the geometric realization functor with values in the category of compactly generated Hausdorff spaces, and apply it to the above diagram. It preserves finite limits by a result of Gabriel and 
Zisman [10], and takes Kan fibrations to Serre fibrations by a result of Quillen [18]. In the topological world, the claim follows from the five lemmas applied to the homomorphism induced between the homotopy long exact sequences of $p$ and $q$; and that implies the conclusion for the simplicial (or 'combinatorial') homotopy groups too.

Proposition 2.2 is a formal consequence of Proposition 2.1. There are three strategies for seeing that:

(1) Both the hypotheses and the desired conclusion of Proposition 2.1 can be phrased purely in the language of sets, membership, ordered tuples and projections, and unions and intersections (allowing countable unions as well), in the syntax known as geometric logic; see the textbook of Moerdijk and MacLane [16]. There is an interpretation of the language of sets in any topos (akin to the formalism of 'virtual elements' and 'diagram chases via virtual elements' in arbitrary abelian categories, cf. [15]). This interpretation, at the same time, defines precisely the meaning of local and ensures - thanks to a theorem of Joyal, Deligne and others, and one of Makkai and Reyes, respectively-that theorems whose hypotheses and conclusions can be phrased in finitary resp. countable geometric logic, stay valid in an arbitrary topos.

(2) Both the hypotheses and the conclusion of Proposition 2.2 can be formulated in the language of diagrams, finite limits and countable colimits. For example, to say that $Y \stackrel{p}{\longrightarrow} B$ is a local Kan fibration in $\varepsilon^{\Delta^{\text {op }}}$ means that the canonical maps $Y_{n} \rightarrow \Lambda_{n}^{k}(Y) \times_{\Lambda_{n}^{k}(B)} B_{n}$ from the object of $n$-simplices of $Y$ to the matching object of $(n, k)$-horns in $Y$ above $n$-simplices of $B$, are epimorphisms. To say that a map in $\varepsilon^{\Delta^{\mathrm{op}}}$

$$
A \stackrel{g}{\longrightarrow} B
$$

induces an epi on $\pi_{n}$ for all local basepoints means that the corner map in the diagram

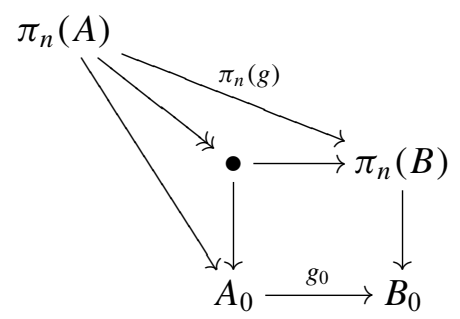

is an epimorphism. Here $A_{0}, B_{0}$ are the degree 0 parts of the simplicial objects $A, B$ ("all the local basepoints"); $\pi_{n}(A), \pi_{n}(B)$ are the bundle of homotopy groups above them (i.e. group objects in $\varepsilon / A_{0}, \varepsilon / B_{0}$ ), and $\bullet$ stands for a pullback. $\pi_{n}(A)$ is, in turn, a certain subquotient of 
the degree $n$ part of $\operatorname{Ex}^{\infty}(A)$, which is a countable colimit of $\operatorname{Ex}^{k}(A)$, each of which, and the connecting maps as well, arises as collections of matching tuples and projection maps...

The data thus packaged - a set of diagrams, together with requirements that given subdiagrams be limits resp. colimits - go by the name of sketch; see [1] or [4] for a full and careful definition. Propositions 2.1 and 2.2 are identical in this form; they both state that if certain subdiagrams of a given sketch are assumed to be limits resp. colimits, then certain other(s) will be limits (colimits) too. The fundamental theorem of sketches states that if a theorem sketchable via finite limits and finite colimits holds in the category Set, then it will hold in any topos; and same holds if the sketch contains countable colimits, but the total cardinality of all diagrams used is countable. (In simplicial homotopy theory, one often needs this second version because of $\mathrm{Ex}^{\infty}$.)

(3) Any Grothendieck topos $\varepsilon$ has a Boolean cover, that is to say, a complete Boolean algebra $\circ$ and a surjective geometric morphism $\mathrm{Sh}(\circ) \stackrel{f}{\longrightarrow}$ $\varepsilon$. $f^{*}$ preserves and reflects statements that can be formalized in geometric logic. Sh(o) provides a Boolean-valued model for the axioms of Zermelo-Fraenkel set theory (including the axiom of choice). Hence if a theorem of geometric logic is provable in $\mathrm{ZFC}$, it holds in the internal logic of every Grothendieck topos.

\subsection{TERMINOLOGY}

The phrase 'for logical reasons', when used in a proof, signifies that one can appeal to any one of the strategies (1), (2) or (3) to verify a claim in $S e t$, in order to conclude its validity in every topos.

\section{3. $n$-Covers}

\subsection{RECOLLECTIONS ON THE COSKELETON FUNCTOR}

Let $\Delta[0, n]$ denote the full subcategory of $\Delta$ (which is the category of finite ordinals and monotone maps) with objects $0,1, \ldots, n$. The truncation functor $S$ Set $=S e t^{\Delta^{\mathrm{op}}} \stackrel{\operatorname{tr}_{n}}{\longrightarrow} \operatorname{Set}^{\Delta[0, n]^{\mathrm{op}}}$ has a right adjoint (a Kan extension) which we will denote by $\operatorname{cosk}_{n}$. (Note that the literature often uses $\operatorname{cosk}_{n}$ or $\operatorname{Cosk}_{n}$ to denote what would be the composite $\operatorname{cosk}_{n} \circ \operatorname{tr}_{n}$ in this paper.)

PROPOSITION 3.1. For $X \in$ SSet, the following are equivalent:

(1) $X$ is isomorphic to an object in the image of $\cos _{n}$.

(2) The canonical morphism $X \rightarrow \operatorname{cosk}_{n} \circ \operatorname{tr}_{n}(X)$ is an isomorphism. 
(3) Write $\partial \Delta_{k}(X)$ for the set of $(k+1)$-tuples of $(k-1)$-simplices of $X$ that are compatible so as to form the boundary of a standard $k$-simplex. The canonical map $X_{k} \stackrel{b_{k}}{\longrightarrow} \partial \Delta_{k}(X)$ (whose coordinates are the boundary mappings) is a bijection for all $k>n$.

(4) The canonical map $X_{k} \rightarrow \operatorname{hom}\left(\operatorname{tr}_{n}\left(\Delta_{k}\right), \operatorname{tr}_{n}(X)\right)$ is a bijection for all $k>n$. (This map sends a $k$-simplex of $X$, thought of as a simplicial map $\Delta_{k} \rightarrow X$, to its $n$-truncation.)

(5) For any $k>n$ and any diagram in the shape of the solid arrows<smiles></smiles>

precisely one lift exists that makes it commutative. (Here $\partial \Delta_{k}$ stands for the $k-1$-skeleton, that is "boundary", of the standard k-simplex $\Delta_{k}$.)

If $X$ satisfies (any, hence all) these conditions, it is said to be $n$-coskeletal.

The first two versions of this definition can be repeated verbatim for simplicial objects in any category with finite limits (in particular, a topos); and also the third, if one uses iterated pullbacks to assemble the 'boundary object' $\partial \Delta_{k}(X)$. From (1) to (3) remain equivalent, and will serve as the definition of coskeletal objects in general. Case (4) is probably the most easily visualizable description of the coskeleton functor for simplicial sets, and property (5) is included for completeness and comparison; cf. the notion of exact fibration below.

\subsection{SIMPLICIAL TORSORS}

Given $X \in \mathcal{E}^{\Delta^{\mathrm{op}}}$, a simplicial torsor over $X$ (in the broadest sense) is a map $T \rightarrow X$ such that $T \rightarrow \mathbf{1}$ is a weak equivalence-i.e. $T$ has locally the weak homotopy type of a 'point', the terminal object of $\varepsilon^{\Delta^{\text {op }}}$. Defining a map of torsors to be a simplicial map over $X$, they form a full subcategory ST $(X)$ of the overcategory $\varepsilon^{\Delta^{\text {op }}} / X$. Write $\pi_{0} \mathcal{C}$ for the class of connected components of a category $\mathcal{C}$.

THEOREM 3.2. There is a natural map $\pi_{0} \mathrm{ST}(X) \rightarrow \mathrm{ho}_{\mathcal{E}^{\Delta^{\mathrm{op}}}}(\mathbf{1}, X)$ which is a bijection if $X$ is locally Kan.

This is a close variant on work of Brown [5] and Jardine [14]. See [3] for a proof.

In the 1970s, Duskin defined a certain class of simplicial objects $X$ for which, as it turns out, a much smaller collection of simplicial torsors $T$ 
suffices to calculate homotopy classes. When one thinks of these torsors as lying over the terminal object instead, they amount to the notion of cover that is the subject matter of this article.

Recall that a map $X \stackrel{f}{\longrightarrow} Y$ of simplicial sets is a Kan fibration, or satisfies Kan's lifting condition in dimension $k$ if in every commutative square of the form

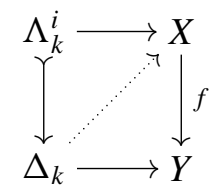

a lift, as indicated by the dotted arrow, exists. $\left(\Lambda_{k}^{i}\right.$ is the simplicial set obtained by omitting the non-degenerate simplex of dimension $k$ and the face opposite the vertex $i, 0 \leqslant i \leqslant k$, from $\Delta_{k}$.) The following definition and proposition are due to Duskin [7].

DEFINITION 3.3. A simplicial map $X \stackrel{f}{\longrightarrow} Y$ satisfies the exact Kan condition in dimension $k$ if in any commutative diagram of the type (3.1), precisely one dotted lift exists. $X \in S S$ et is an exact $n$-type if $X \rightarrow \mathbf{1}$ is a Kan fibration which is exact in dimension $k$ for all $k>n$.

Another way to phrase the condition of $X$ being an exact $n$-type is: the canonical map $X_{k} \rightarrow \Lambda_{k}^{i}(X)$ from the object of $k$-simplices to the object of $(k, i)$-horns is epi for $k \leqslant n$ and an isomorphism for $k>n$. For logical reasons, this formulation is valid in any topos. (In other words, for a topos $\varepsilon$, $X \in \mathcal{E}^{\Delta^{\mathrm{op}}}$ is an exact $n$-type if $X \rightarrow \mathbf{1}$ is a local Kan fibration which is exact above dimension $n$; the notion of 'exact' has a unique meaning, no local and global versions.)

PROPOSITION 3.4. Let $\mathcal{E}$ be a topos, $T \in \mathcal{E}^{\Delta^{o p}}$.

(i) If $T$ satisfies the exact Kan condition above dimension $n$, then $T$ must be $n+1$-coskeletal.

(ii) If $T$ is $n$-coskeletal, then it satisfies the exact Kan condition above dimension $n+1$.

(iii) If $T$ is locally Kan and $n$-coskeletal, then it has vanishing local homotopy groups in dimension $n$ and above.

(iv) An exact n-type has vanishing local homotopy groups above dimension $n$.

Proof. For logical reasons, it suffices to prove these for $\mathcal{E}=$ Set. 
(i) Suppose one has $n+3$ matching $n+1$-simplices that together can be assembled into an $n+2$-boundary in $T$, i.e. a map $\partial \Delta_{n+2} \stackrel{b}{\longrightarrow} T$. Omitting (say) the 0th of these simplices, one obtains a horn $\Lambda_{n+2}^{0} \hookrightarrow$ $\partial \Delta_{n+2} \rightarrow T$. By assumption, this horn has a filler $\Delta_{n+2} \stackrel{f}{\longrightarrow} T$. Consider the two $n+1$-simplices: $d_{0} \Delta_{n+2} \hookrightarrow \Delta_{n+2} \stackrel{f}{\longrightarrow} T$ (the 0th face of $f$ ) and the 0th simplex in the matching set $\partial \Delta_{n+2} \stackrel{b}{\longrightarrow} T$. They have the same boundary; since $T$ satisfies the exact Kan condition above dimension $n$, they must coincide. So $f$ is a filler for $b$. Again by exactness in dimension $n+2$, this filler must be unique.

(ii) By adjunction, $\operatorname{cosk}_{n}(X)$ satisfies the exact Kan condition with respect to $\Lambda_{m}^{k} \longmapsto \Delta_{m}$ iff $X$ satisfies the unique lifting condition with respect to $\operatorname{tr}_{n}\left(\Lambda_{m}^{k} \longmapsto \Delta_{m}\right)$. That will certainly happen for $m>n+1$, for $\operatorname{tr}_{n}\left(\Lambda_{m}^{k} \longmapsto \Delta_{m}\right)$ is an isomorphism then. (Note that $\Lambda_{m}^{k}$ leaves out cells of dimension $m-1$ and higher from $\Delta_{m}$.)

(iii) A 0-coskeletal simplicial set is just the nerve of a groupoid that is equivalent (as a category) to the terminal category; so it is automatically Kan, and (if non-empty) simplicially contractible.

If $n>0$, choose any 0 -simplex $x \in T_{0}$ and any $k$-simplex $y \in T_{k}, k \geqslant n$, all of whose faces are (the degeneracies of) $x$. This $y$, together with $k+1$ copies of the (unique) $k$-dimensional degeneracy of $x$, make up a compatible boundary of a $k+1$-simplex. Since $T$ is $n$-coskeletal, this boundary has a filler. Thence $y$ is null-homotopic modulo its boundary. Since $T$ was assumed to be Kan, this means its homotopy groups are trivial in dimension $k$, any $k \geqslant n$.

(iv) By definition, an exact $n$-type is a Kan complex; now use (i) and (iii).

\subsection{ASIDES ON EXACT $n$-TYPES}

The following observation of Duskin [7] must have been motivational in his definition of 'exact': let $n \in \mathbb{N}$ and $A$ be an abelian group (just a group if $n=1$, or a set if $n=0)$. Then $K(A, n)$, the standard simplicial model of an Eilenberg-MacLane space, is an exact $n$-type.

By the standard model we mean the constant simplicial set $A$ if $n=0$, the nerve of $A$ if $n=1$, and the de-normalization of the appropriate chain complex concentrated in degree $n$ if $n>1$. Recall that this $K(A, n)$ is $n$-reduced, i.e. has a singleton in dimensions below $n, A$ itself in dimension $n$, suitable tuples of elements of $A$ in dimension $n+1$, and is coskeletal above that.

Duskin [7] actually introduced the term n-hypergroupoid for what is called exact $n$-type here, while Glenn [11] uses ' $n$-hypergroupoid' to mean 
any simplicial set satisfying the exact Kan condition above dimension $n$ (not requiring it to be a Kan complex in all dimensions). I confess to replacing ' $n$-hypergroupoid' by 'exact $n$-type' (prompted by, of course, Duskin's definition of 'exact Kan fibration', Definition 3.3) so as to emphasize its homotopical meaning (valid for all $n$ ) and de-emphasize its link to groupoids (known to be valid for small $n$ ). In this context, one should also observe that the exact Kan condition above dimension $n$ places no restriction on the homotopy type of $X$ in the absence of the Kan condition in lower dimensions. For example, the nerve of any small category is 2-coskeletal, hence satisfies the exact Kan filling condition in dimensions greater than 3. Nonetheless, any homotopy type can be realized as the nerve of a small category. By way of contrast, if the nerve of a category is a Kan complex (which means, therefore, extra conditions only in dimensions 1-3), then that category is a groupoid, and its nerve has the homotopy type of a disjoint union of Eilenberg-MacLane spaces.

- The converse to Proposition 3.4(iv) holds as well, i.e. every (topological) $n$-type has simplicial models that are exact $n$-types. In fact, we will see in Proposition 3.5 that there is a model for the Postnikov section functor that takes values in exact $n$-types.

- Duskin [8] describes a fascinating example of a non-abelian exact 3-type, the Azumaya complex $\mathrm{AZ}(R)$ of a commutative ring $R . \mathrm{AZ}(R)$ has a unique vertex $*$; its edges are Azumaya $R$-algebras, its 2 -simplices certain invertible bimodules and its 3 -simplices, isomorphisms between those. $\pi_{i}(\mathrm{AZ}(R), *)$ is isomorphic to the Brauer group of $R$ (defined as equivalence classes of Azumaya algebras), the Picard group of $R$, and $R^{\times}$, respectively, for $i=1,2,3$.

One surmises that more constructions of this type exist in the domains of deformation theory, formal group laws or bordism-though as $n$ increases, it requires more and more ingenuity to make 'deformation data of the $n$th order' fit the geometry of $n$-simplices.

- Note that an exact $n$-type is also an exact $m$-type for any $m>n$. An exact $n$-type is a minimal simplicial set (in the sense of Kan) in dimensions $k \geqslant n$, meaning that two $k$-simplices that are homotopic modulo their boundaries must be equal, but this need not hold in dimensions below $n$. Consequently, exact $n$-types are not necessarily economical models of topological $n$-types; as we will see, there are infinitely many exact $n$-type models of the point(!), and they can be quite complicated.

\subsection{DUSKIN-POSTNIKOV SECTIONS}

Duskin has also defined a particularly good model for Postnikov sections. His results appear in [11]; we only summarize the facts needed later. 
PROPOSITION 3.5. For each $n \in \mathbb{N}$, there exists a functor $\mathcal{P}_{n}$ from the full subcategory of simplicial sets whose objects are Kan complexes to its full subcategory with objects the exact n-types, as well as a natural transformation $p_{n}$ from the identity to $\mathcal{P}_{n}$ with the following properties:

- $\mathcal{P}_{n}$ preserves Kan fibrations,

- $p_{n}$ induces isomorphisms on $\pi_{i}$ for $i \leqslant n$,

- $\mathcal{P}_{n}$ and $p_{n}$ are definable in terms of finite limits and countable colimits.

We can now state our main definition and its most important property.

DEFINITION 3.6. An $n$-cover of a topos $\mathcal{E}$ is an exact $n$-type $T \in \mathcal{E}^{\Delta^{\text {op }}}$ such that $T \rightarrow \mathbf{1}$ is a local weak equivalence.

PROPOSITION 3.7. Let $\mathcal{E}$ be a topos and $X \in \mathcal{E}^{\Delta^{o p}}$ an exact $n$-type. Write $\operatorname{cov}_{\mathcal{E}}^{n}(X)$ for the full subcategory of $\mathrm{ST}(X)$ with objects $T \rightarrow X$ such that $T$ is an $n$-cover of $\mathcal{E}$. Then there is a canonical bijection $\pi_{0} \operatorname{cov}_{\mathcal{E}}^{n}(X) \rightarrow$ ho $_{\mathcal{E}^{\Delta^{o p}}}(\mathbf{1}, X)$.

Proof. The bijection is $\pi_{0} \operatorname{cov}_{\mathcal{E}}^{n}(X) \rightarrow \pi_{0} \mathrm{ST}(X) \rightarrow \mathrm{ho}_{\mathcal{E}^{\Delta \mathrm{op}}}(\mathbf{1}, X)$, where the first map is induced by $\operatorname{cov}_{\mathcal{E}}^{n}(X) \hookrightarrow \mathrm{ST}(X)$ and the second map is that of Theorem 3.2. So it suffices to prove the first one to be a bijection.

Now quite generally, if one has two categories $\mathcal{C}, \mathcal{D}$ and functors $\mathcal{C} \stackrel{F}{\longrightarrow} \mathcal{D}, \mathcal{C} \stackrel{G}{\longleftarrow} \mathcal{D}$ such that every object $c$ of $\mathcal{C}$ is connected to $G F(c)$ via a zig-zag of arrows in $\mathcal{C}$, and every object $d$ of $\mathcal{D}$ is connected to $F G(d)$ via a zig-zag of arrows in $\mathcal{D}$ (these zig-zags are not required to be functorial, or even be of some fixed shape) then $\pi_{0} F$ and $\pi_{0} G$ are inverse bijections between $\pi_{0} \mathcal{C}$ and $\pi_{0} \mathcal{D}$. It follows that if $F$ is onto on hom-sets (for example, it is the inclusion of a full subcategory) and for any $d$ in $\mathcal{D}$ there exists a map $d \rightarrow F G(d)$, then $F$ and $G$ induce bijections on connected components. Apply this to the full inclusion $\operatorname{cov}_{\mathcal{E}}^{n}(X) \stackrel{F}{\longrightarrow} \mathrm{ST}(X), G$ being defined by the diagram

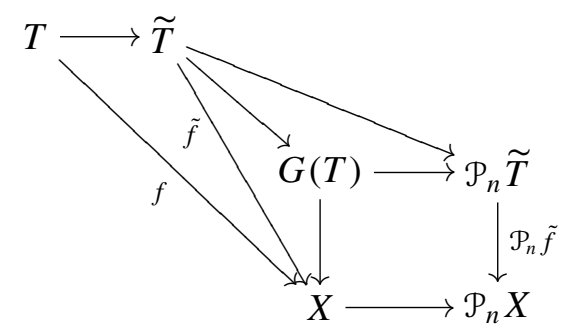

Starting with a torsor $T \stackrel{f}{\longrightarrow} X$, factor it (functorially) as a weak equivalence $T \rightarrow \widetilde{T}$ followed by a local fibration $\widetilde{T} \stackrel{\tilde{f}}{\longrightarrow} X$. Apply $\mathcal{P}_{n}$ to $\tilde{f}$, 
$\widetilde{T} \rightarrow \mathcal{P}_{n} \widetilde{T}$ and $X \rightarrow \mathcal{P}_{n} X$ coming from the natural transformation $p_{n}$ of Proposition 3.5, and let $G(T)$ be the indicated pullback, the (natural) map $T \rightarrow G(T)=F G(T)$ (over $X$ ) being the above composite.

First, $\mathcal{P}_{n} \tilde{f}$ is a local fibration, and so is its pullback $G(T) \rightarrow X$; since $X$ is locally Kan, so is $G(T)$. Second, $G(T)$, being a pullback of three objects each of which satisfies the exact Kan condition above dimension $n$, itself satisfies it; so it is an exact $n$-type, and has vanishing local homotopy groups above $n$. Finally, $\mathcal{P}_{n} \widetilde{T}$, being a Postnikov section of an object that is weakly equivalent to 1 , is itself weakly equivalent to $1 . G(T) \rightarrow \mathcal{P}_{n} \widetilde{T}$ is an $n$-equivalence by virtue of Proposition 2.2; so $G(T)$ has trivial local homotopy groups up to dimension $n$ too. Therefore $G(T) \rightarrow X$ belongs to $\operatorname{cov}_{\mathcal{E}}^{n}(X)$, and we have a functor and natural transformation as required.

\subsection{SCHOLIUM}

There are several functorial models of the Postnikov $n$-section of a Kan complex $X$, the simplest being, no doubt, $\operatorname{cosk}_{n+1} \circ \operatorname{tr}_{n+1}(X)$. Some version of the main theorem would work with each; the difference is that they would not specialize to the classical theory of Čech covers. See Remark 4.7 for an elaboration of this point. As regards Duskin's $\mathcal{P}_{n}$, though all we need is contained in Propostion 3.5, it may be worthwhile to compare the details of his construction with the much better known model due to Moore (see e.g. [13], Section VI.3).

The Moore-Postnikov $n$-section $P_{n}^{M}(X)$ is the image of the canonical map $X \rightarrow \operatorname{cosk}_{n} \circ \operatorname{tr}_{n}(X)$. This is the same as saying that $P_{n}^{M}(X)$ is a quotient complex of $X$, where two $k$-simplices $x, y \in X_{k}$ are identified if they have identical boundaries up to dimension $n$, i.e. identical $n$-truncations.

For the Duskin-Postnikov $n$-section $\mathcal{P}_{n}(X)$, consider the truncation $\operatorname{tr}_{n}(X)$ first. Introduce a relation $\sim_{n}$ on its $n$-simplices by defining two to be equivalent if they are simplicially homotopic in $X$ modulo their boundaries. (This is an equivalence relation by virtue of the assumption that $X$ is a Kan complex.) Since the face maps from $X_{n}$ factor through this equivalence, there results a well-defined truncated simplicial object $\operatorname{tr}_{n}(X) / \sim_{n}$. (It is not being claimed that $X / \sim_{n}$ would be a well-defined simplicial object!) There is a canonical map $X \stackrel{p}{\longrightarrow} \operatorname{cosk}_{n}\left(\operatorname{tr}_{n}(X) / \sim_{n}\right)$, adjoint to the quotient map $\operatorname{tr}_{n}(X) \rightarrow \operatorname{tr}_{n}(X) / \sim_{n}$. Define $\mathcal{P}_{n}(X)$ to be the image of $p$.

$\mathcal{P}_{0}(X)$ is the disjoint union of $\pi_{0}(X)$ many copies of the (simplicial) point, the terminal object of SSet. $P_{0}^{M}(X)$ is the disjoint union of $\pi_{0}(X)$ many simplicially contractible sets; these are of the form $\operatorname{cosk}_{0}\left(X_{0, i}\right)$, where $X_{0, i}$ is the vertex set of the $i$ th connected component of $X$.

$\mathcal{P}_{1}(X)$ is the nerve of $\pi_{1}(X)$, the Poincare groupoid of $X$ (one object for each 0 -simplex and one edge for each homotopy class of 1 -simplices). 
$P_{1}^{M}(X)$ keeps all the 0 -simplices and all the 1 -simplices of $X$. It is not the nerve of any category (though it is simplicially homotopy equivalent to $N \pi_{1}(X)$, of course).

In short, $\mathcal{P}_{n}(X)$ is a 'slimmer' and geometrically perhaps more natural Postnikov section, even though the extra reduction only involves the $n$-simplices. It also has the beautiful property (which we did not use) that it is idempotent (as opposed to just homotopy idempotent) on locally Kan objects in $\mathcal{E}^{\Delta^{\mathrm{op}}}$. Note finally that $P^{M}(X)$ and $\mathcal{P}(X)$ need not have the intended homotopy type, and certainly need not be (local) Kan complexes, unless $X$ is a (local) Kan complex.

\subsection{FINITARY DESCRIPTION OF EXACT $N$-TYPES AND $n$-COVERS}

The notions of exact fibration, exact $n$-type and $n$-cover are preserved by inverse image parts of topos morphisms, in particular, by stalk functors. In the case of toposes with enough points, these notions are also reflected by stalk functors (for the usual logical reasons), e.g. $T \in \mathcal{E}^{\Delta^{\text {op }}}$ will be an $n$-cover iff all stalks of $T$ are $n$-covers in SSet.

Definition 3.6, after a little unwrapping, turns out to describe structures well known in low dimensions. We will decode these first.

\subsection{0-COVERS}

An exact 0-type in SSet is a simplicial set that is constant, i.e. all of whose structure maps are the identity. A topos $\mathcal{E}$ has (up to isomorphism) a single 0 -cover, the constant simplicial object on the terminal object of $\mathcal{\varepsilon} \cdot \operatorname{cov}_{\mathcal{E}}^{0}$ is equivalent to the trivial (unique object, only the identity morphism) category.

\subsection{1-COVERS}

An exact 1-type $T \in S S e t$ turns out to be precisely the same as the nerve of a groupoid - see [7]. The graph underlying this groupoid is just the 1-truncation $T_{1} \rightrightarrows T_{0}$ of $T$. Such a simplicial set $T$ has the homotopy type of a point iff the corresponding groupoid has one component and trivial vertex groups, which happens iff $T$ is 0 -coskeletal and non-empty. Correspondingly, a 1 -cover of a topos $\mathcal{E}$ is a 0 -coskeletal simplicial object $X \Leftarrow$ $X^{2} \Leftarrow X^{3} \cdots$ such that $X \rightarrow \mathbf{1}$ is an epimorphism. A simplicial morphism between 0 -coskeletal objects is necessarily induced from an ordinary map at level 0 ; we thus have an adjoint equivalence

$$
\operatorname{cov}_{\mathcal{E}}^{1} \underset{\operatorname{tr}_{0}}{\stackrel{\operatorname{cosk}_{0}}{\leftrightarrows}}\{X \in \mathcal{E} \mid X \rightarrow \mathbf{1}\}
$$


between 1-covers of $\mathcal{E}$ and the full subcategory of $\mathcal{E}$ of objects with global support, that is, ones that map to the terminal object via an epimorphism.

\subsection{2-COVERS}

The reader sceptical of the intricacies of 'higher dimensional diagram chases' is invited to work out a finitary algebraic description of exact 2-types. Duskin's [9] solution is what he calls a bigroupoid, which is a bicategory in which all 1-arrows are equivalences and all 2-arrows are isomorphisms. A bicategory itself (not needed in this paper) is a higher categorical structure made up of objects, 1-arrows and 2-arrows, such that the composition of 1-arrows is not associative; its failure to be associative is measured by a (functorially assigned) 2-isomorphism, which in turn is subject to a coherence condition; this coherence condition can be visualized as a 4-simplex, or alternatively as a MacLane-Stasheff pentagon. Identities are likewise relaxed. Duskin assigns a simplicial set, a 'nerve', to any bicategory - this being a rather more involved affair than the nerves of strict higher categories - and proves that the nerve is an exact 2-type iff it arises from a bigroupoid.

What of 2-covers, that is, exact 2-types weakly equivalent to a point? It follows from general principles - see Proposition 3.10 - that a 2-cover, as a simplicial object, must be 1-coskeletal. Let us take $\mathcal{E}=$ Set, and think of the truncation of a simplicial set to levels 0 and 1 as a graph $T_{1} \underset{t}{\stackrel{s}{\rightrightarrows}} T_{0}$; 'graph' will mean 'directed graph equipped with unit arrows at each vertex' throughout, but we will omit notation for units for the sake of legibility.

Since $\pi_{0}$ of the 1-coskeleton of this graph must be trivial, $T_{0}$ must be non-empty and the graph must be connected. The Kan fibrancy condition on its 1-coskeleton implies that our graph satisfies the right lifting condition (or 'injectivity condition'), in the category of graphs, with respect to the following three inclusions, which should be thought of as the edge truncations of the three Kan horn conditions in dimension 2

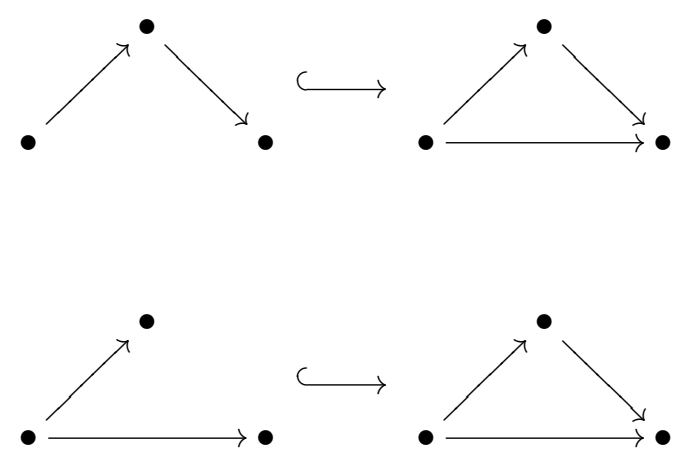




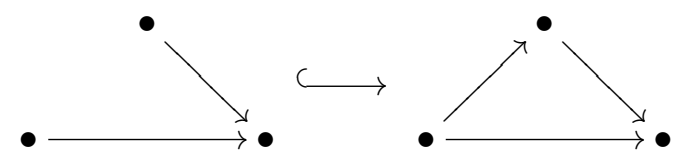

But given that the graph is connected, these imply that for every pair $u, v$ of vertices, there is some edge starting at $u$ and ending at $v$. Conversely, this latter condition implies that $\operatorname{cosk}_{1}\left(T_{1} \underset{t}{\stackrel{s}{\rightrightarrows}} T_{0}\right)$ satisfies the Kan condition in dimension 2 (as well as, tautologically, in dimension 1); from being 1-coskeletal, it satisfies the Kan condition exactly above dimension 2; it is connected, and its $\pi_{n}$ vanishes for $n \geqslant 1$, by virtue of being Kan and 1-coskeletal. To conclude, a 2-cover in SSet is the same data as a graph $T_{1} \underset{t}{\stackrel{s}{\rightrightarrows}} T_{0}$ such that $T_{0}$, the set of vertices, is non-empty, and $T_{1} \stackrel{s \times t}{\rightarrow} T_{0} \times T_{0}$ is surjective. The 2-cover associated to this data is just the 1-coskeleton of the truncated simplicial object $T_{1} \underset{t}{\stackrel{s}{\rightrightarrows}} T_{0}$.

For logical reasons, one actually has

COROLLARY 3.8. There is an adjoint equivalence

$$
\operatorname{cov}_{\mathcal{E}}^{2} \underset{\operatorname{tr}_{1}}{\stackrel{\text { oosk }_{1}}{\leftrightarrows}}\left\{\text { diagrams } T_{1} \underset{t}{\stackrel{s}{\rightrightarrows}} T_{0} \text { in } \mathcal{E}\right. \text { such that (0) and (1) \} }
$$

between 2-covers of $\mathcal{E}$ and the full subcategory of graph objects in $\mathcal{E}$ satisfying

(0) $T_{0} \rightarrow \mathbf{1}$ is an epimorphism,

(1) $T_{1} \stackrel{s \times t}{\rightarrow} T_{0} \times T_{0}$ is an epimorphism.

This is also a special case of Proposition 3.11.

Remark 3.9. If one prefers the bigroupoid formalism, then one can think of the injectivity condition with respect to (3.2) as expressing the possibility of composing graph edges, and (3.3) and (3.4) resp. as permitting 'left' and 'right' division, without assuming that such operations have been chosen, or indeed, that they can be chosen with any kind of consistency. The bigroupoid associated to the data $T_{1} \rightrightarrows T_{0}$ in Set has $T_{0}$ as objects, $T_{1}$ as 1-arrows, and exactly one 2 -arrow from any 1 -arrow to any other with the same source and target. This also fixes the composition of 2-arrows. To define composition of 1-arrows, choose, completely arbitrarily, a composite for each composable pair, respecting source and target - this is made possible by (3.2). The association isomorphism, being a 2-arrow between the 1 -arrows $(a b) c$ and $a(b c)$, is uniquely defined. The coherence condition on the next level, which requires that the two possible re-association sequences 
from $(((a b) c) d$ to $a(b(c d)))$, when expressed as 2-arrows, coincide, is satisfied tautologously. In this bicategory, obviously every 1-arrow is an equivalence and every 2-arrow an isomorphism; so it is a bigroupoid.

The correspondence between bigroupoids and exact 2-types breaks down when one passes to sheaves of them, since the requisite choices can be made locally at best. A bigroupoid does carry more information than an exact 2-type; for example, functional choices of fillers for certain horns. Equivalently, the category of bigroupoids (as a many-sorted universal algebra) will not be naturally equivalent to the category of exact 2-types (as full subcategory of $S S e t$ ). In this paper, we will employ direct simplicial descriptions throughout.

\subsection{0. n-COVERS}

These turn out to be, quite simply, truncated simplicial objects with simplex filling conditions up to the top dimension.

PROPOSITION 3.10. If $T$ is an $n$-cover, then it is $(n-1)$-coskeletal.

Proof. For logical reasons, it is enough to prove this for simplicial sets. By Proposition 3.1, what we have to prove is that the canonical maps $T_{k} \stackrel{b_{k}}{\longrightarrow} \partial \Delta_{k}(T)$ are bijections for $k>n-1$.

Recall that in SSet, a Kan complex $T$ that has the weak homotopy type of the point will satisfy the right lifting condition with respect to the boundary inclusions, meaning the $b_{k}$ will be surjective for all $k$. (For logical reasons, this second, 'local' version remains valid in any topos.) By virtue of $T$ being exact Kan above dimension $n, b_{k}$ will be injective for $k>n$ (if any $k$-horn has precisely one filler, then certainly any $k$-boundary can have at most one filler). Thence $b_{k}$ is bijective for $k>n$.

So we are left to prove that $b_{n}$ is injective. It is easiest to argue topologically. Let $\Delta_{n} \stackrel{s}{\longrightarrow} T, \Delta_{n} \stackrel{t}{\longrightarrow} T$ be two $n$-simplices of $T$ whose boundaries coincide. Since $T$ was assumed to have the weak homotopy type of a point, the geometric realizations of $s$ and $t$ are homotopic modulo the boundary. Since $T$ was assumed to be a Kan complex, $s$ and $t$ are simplicially homotopic modulo the boundary. But two $n$-simplices of an exact $n$-type that are simplicially homotopic modulo the boundary, must coincide.

PROPOSITION 3.11. Write $\Delta[0, n-1]$ for the full subcategory of $\Delta$ (i.e. the category of finite ordinals and monotone maps) whose objects are 0, 1, $\ldots, n-1$. There is an adjoint equivalence

$$
\operatorname{cov}_{\mathcal{E}} \underset{\operatorname{tr}_{n-1}^{n}}{\stackrel{\operatorname{cosk}_{n-1}}{\leftrightarrows}}\left\{T \in \mathcal{E}^{\Delta[0, n-1]^{o p}} \mid(\star) \text { holds for } k=0,1,2, \ldots, n-1\right\}
$$


between $n$-covers of $\mathcal{E}$ and the full subcategory of $n$-1-truncated simplicial objects in $\varepsilon$ satisfying

( $\star T_{k} \stackrel{b_{k}}{\rightarrow} \partial \Delta_{k}(T)$ is an epimorphism

where $\partial \Delta_{k}(T)$ and $b_{k}$ are defined as in Proposition 3.1(3).

Proof. Use, again, that a simplicial object $T$ in a topos has the weak homotopy type of the point and is locally Kan at the same time if and only if the maps $T_{k} \stackrel{b_{k}}{\rightarrow} \partial \Delta_{k}(T)$ are epimorphisms for all $k$. The part of the claim that $\operatorname{tr}_{n-1}$ is a functor between these two categories follows from the 'only if' direction of this. That $\operatorname{cosk}_{n-1}$ is a functor with the desired properties follows from the 'if' direction, together with Proposition 3.4(ii). The adjoint equivalence follows from Proposition 3.10.

As in any simplicial category with finite limits and colimits, there is a notion of simplicial homotopy in $\mathcal{E}^{\Delta^{\mathrm{op}}}$ : the role of the interval is played by the constant simplicial sheaf $\Delta[1]$ with its two global sections corresponding to $\Delta[0] \stackrel{i_{0}, i_{1}}{\longrightarrow} \Delta[1]$. Post- and pre-composition of maps respect simplicial homotopies. Write $[X, Y]$ for the hom-set $\operatorname{hom}_{\mathcal{E}^{\Delta}}$ op $(X, Y)$ modulo the equivalence relation generated by simplicial homotopy. Let $\operatorname{cov}_{\mathcal{E}}^{n}$ be the full subcategory of $\varepsilon^{\Delta^{o p}}$ whose objects are $n$-covers, and write $\left[\operatorname{cov}_{\varepsilon}^{n}\right]$ for the category with the same objects, but morphisms from $T_{1}$ to $T_{2}$ being $\left[T_{1}, T_{2}\right]$.

PROPOSITION 3.12. $\left[\operatorname{cov}_{\mathcal{E}}^{n}\right]$ is a cofiltered category.

Proof. The product of two $n$-covers is an $n$-cover. The possibility of equalizing (in the simplicial homotopy category) a parallel pair of arrows between $n$-covers follows, given Proposition 3.11, from the proof of the analogous fact for Verdier hypercovers as given by Artin and Mazur [2].

A hypercover of $\mathcal{E}$ is, by definition, a full simplicial object $T \in \mathcal{E}^{\Delta^{\mathrm{op}}}$ such that $T_{k} \stackrel{b_{k}}{\rightarrow} \partial \Delta_{k}(T)$ is an epimorphism for all $k \in \mathbb{N}$. Given parallel arrows $T_{1} \rightrightarrows T_{2}$ between two hypercovers, Artin and Mazur construct a hypercover $T$, map $T \rightarrow T_{1}$ and simplicial homotopy $T \times \Delta[1] \rightarrow T_{2}$ by induction, working up degree-by-degree. Their construction remains valid, unchanged, up to degree $n$, producing an $n$-truncated simplicial equalizer of a pair of arrows between $n$-truncated simplicial objects that satisfy the boundary-filling conditions up to degree $n$.

PROPOSITION 3.13. $\left[\operatorname{cov}_{\mathcal{E}}^{n}\right]$ possesses (non-canonical) small cofinal subcategories. 
Proof. $\operatorname{cov}_{\mathcal{E}}^{n}$ is an accessible category in the sense of Makkai and Paré [17], either directly from its definition in geometric logic or because, by virtue of Proposition 3.11, it is the category of models of a sketch (see Section 2 for details of this notion). It follows that it has a small dense subcategory $\mathcal{G} \hookrightarrow \operatorname{cov}_{\mathcal{E}}^{n}$; meaning that every $n$-cover is a filtered colimit of a diagram from $\mathcal{G}$, a fortiori every $n$-cover permits at least one map from a member of $\mathcal{G}$. The image of $\mathcal{G}$ in $\left[\operatorname{cov}_{\mathcal{E}}^{n}\right]$ is a full cofinal small subcategory.

PROPOSITION 3.14. Write hom $(-, X)$ resp. $[-, X]$ for the contravariant hom- resp. hom- modulo simplicial homotopy functors $\mathcal{E}^{\Delta^{o p}} \rightarrow$ Set. Each of

$$
\begin{aligned}
& \underset{\operatorname{cov}_{\varepsilon}^{n}}{\operatorname{colim}} \operatorname{hom}(-, X), \\
& \operatorname{colim}_{\operatorname{cov}_{\varepsilon}^{n}}[-, X], \\
& \underset{\left.\operatorname{colim}_{\varepsilon}^{n}\right]}{\operatorname{col}^{n}}[-, X],
\end{aligned}
$$

bijects canonically with $\pi_{0} \operatorname{cov}_{\mathcal{E}}^{n}(X)$.

Proof. This follows from elementary manipulations on representatives of these colimits.

Recall from Proposition 3.7 that $\operatorname{cov}_{\varepsilon}^{n}(X)$ stands for the category whose objects are $T \rightarrow X, T$ some $n$-cover of $\mathcal{E}$, and whose morphisms are commutative triangles. Write $C_{1}(X)$ for the category whose objects are simplicial homotopy classes of maps $T \rightarrow X, T$ some $n$-cover, a morphism from $T_{1} \rightarrow X$ to $T_{2} \rightarrow X$ being an actual map $T_{1} \rightarrow T_{2}$ that makes the resulting triangle commute up to simplicial homotopy; and write $C_{2}(X)$ for the category whose objects are simplicial homotopy classes of maps $T \rightarrow X, T$ some $n$-cover, and where a morphism from $T_{1} \rightarrow X$ to $T_{2} \rightarrow X$ is a simplicial homotopy class of maps $T_{1} \rightarrow T_{2}$ that makes the resulting triangle commute up to simplicial homotopy. Then

$$
\begin{aligned}
\pi_{0} \operatorname{cov}_{\varepsilon}^{n}(X) & =\underset{\operatorname{cov}_{\varepsilon}^{n}}{\operatorname{colim}} \operatorname{hom}(-, X), \\
\pi_{0} C_{1}(X) & =\operatorname{colim}_{\operatorname{cov}_{\varepsilon}^{n}}[-, X], \\
\pi_{0} C_{2}(X) & =\underset{\left[\operatorname{cov}_{\varepsilon}^{n}\right]}{\operatorname{colim}_{1}}[-, X] .
\end{aligned}
$$

The 'take its simplicial homotopy class' functors $\operatorname{cov}_{\mathcal{E}}^{n}(X) \rightarrow C_{1}(X) \rightarrow C_{2}(X)$ induce surjections $\pi_{0} \operatorname{cov}_{\mathcal{E}}^{n}(X) \rightarrow \pi_{0} C_{1}(X) \rightarrow \pi_{0} C_{2}(X)$. To prove $\pi_{0} \operatorname{cov}_{\mathcal{E}}^{n}(X) \rightarrow$ $\pi_{0} C_{1}(X)$ injective, it suffices to show that if two maps $T \underset{g}{\stackrel{f}{\rightrightarrows}} X$ are simplicially homotopic, then $T \stackrel{f}{\longrightarrow} X$ and $T \stackrel{g}{\longrightarrow} X$ are connected by a zig-zag 
of morphisms in $\operatorname{cov}_{\mathcal{E}}^{n}(X)$. Let $T \times \Delta[1] \stackrel{h}{\longrightarrow} X$ be the homotopy. Note that $T \times \Delta[1]$ is a simplicial object over $X$ that is weakly equivalent to $\mathbf{1}$. As in the proof of Proposition 3.7, $h$ can be factored $T \times \Delta[1] \stackrel{h_{1}}{\longrightarrow} G(T) \stackrel{h_{2}}{\longrightarrow} X$, where $G(T)$ is an $n$-cover. The requisite zig-zag is the composite $T \stackrel{i_{0}}{\longrightarrow}$ $T \times \Delta[1] \stackrel{h_{1}}{\longrightarrow} G(T)$ together with $T \stackrel{i_{1}}{\longrightarrow} T \times \Delta[1] \stackrel{h_{1}}{\longrightarrow} G(T)$. Two applications of this argument show that $\pi_{0} \mathcal{C}_{1}(X) \rightarrow \pi_{0} C_{2}(X)$ is injective.

Propositions 3.7, 3.12, 3.13 and 3.14 together yield our main result. Recall that an $n$-cover of the Grothendieck topos $\mathcal{E}$ is a simplicial object $U \in \mathcal{E}^{\Delta^{\mathrm{op}}}$ that has the weak homotopy type of $\mathbf{1}$, is locally Kan in all degrees and satisfies the unique Kan extension condition above degree $n$, and we write $[-,-]$ for simplicial homotopy classes and $\mathrm{ho}_{\mathcal{E}^{\mathrm{L}^{\mathrm{op}}}}(-,-)$ for global homotopy classes.

THEOREM 3.15. For any $X \in \mathcal{E}^{\Delta^{o p}}$, there is a natural map

$$
\underset{\left[\operatorname{cov}_{\varepsilon}^{n}\right]}{\operatorname{colim}}[-, X] \rightarrow \text { ho }_{\mathcal{E}^{\Delta o p}}(\mathbf{1}, X),
$$

where $\left[\operatorname{cov}_{\varepsilon}^{n}\right]$ is the filtered, essentially small diagram of simplicial homotopy classes of $n$-covers of $\mathcal{E}$. This map is bijective when $X$ is a locally Kan object that satisfies the unique Kan extension condition above degree $n$.

For the sake of completeness, let us include the classical case of ' $n=\infty$ ' here:

PROPOSITION 3.16. A Verdier cover or hypercover of a topos $\mathcal{E}$ is a simplicial object $U \in \mathcal{E}^{\Delta^{o p}}$ that has the weak homotopy type of $\mathbf{1}$ and is locally Kan in all degrees. For any $X \in \mathcal{E}^{\Delta^{o p}}$, there is a natural map

$$
\underset{\left[\operatorname{cov}_{\varepsilon}\right]}{\operatorname{colim}}[-, X] \rightarrow \operatorname{ho}_{\mathcal{E}^{\Delta o p}}(\mathbf{1}, X),
$$

where $\left[\operatorname{cov}_{\varepsilon}\right]$ is the filtered, essentially small diagram of simplicial homotopy classes of hypercovers of $\mathcal{E}$. This map is bijective when $X$ is locally Kan.

\section{4. Čech n-Covers}

This section describes how Theorem 3.15 specializes, for $n=1$, to the traditional formalism of 'open covers and refinements' and outlines the analogous version for $n>1$.

EXAMPLE 4.1. Let $X$ be a topological space. For convenience, we make no notational distinction between spaces étale over $X$ and the sheaves on $X$ they represent, and we talk of ' $n$-covers of $\operatorname{Sh}(X)$ ' as ' $n$-covers of $X$ '. 
(1) Let $\left\{U_{i} \mid i \in I\right\}$ be an open cover of $X$ in the usual sense. Each $U_{i}$ represents, as an étale space over $X$, a subobject of the terminal object 1 of $\operatorname{Sh}(X)$. Set $U$ to be the coproduct of the $U_{i}$ considered as objects of $\operatorname{Sh}(X)$ (equivalently, take their disjoint union as spaces over $X) . U \rightarrow \mathbf{1}$ then, so $\operatorname{cosk}_{0}(\mathcal{U})$ is a 1 -cover of $X$.

(2) Let $C \stackrel{p}{\longrightarrow} X$ be a covering space of $X$ in the usual sense. Then $p$ is certainly étale and surjective over $X$, so $\operatorname{cosk}_{0}(C)$ is a 1 -cover of $X$.

(3) One can have a mixture of (1) and (2): a different covering space over each element of an open cover of $X$. These (and in fact all 1-covers of a space) are dominated by trivializations, thus Čech covers as in (1).

Note that there is a proper class of non-isomorphic 1-covers of $X$, while there are certainly no more open covers of $X$ than collections of open subsets of $X$. Nonetheless, open covers are cofinal among 1-covers. This is a general phenomenon, as we will see.

Let $(\mathcal{C}, J)$ be a site; assume $\mathcal{C}$ has a terminal object, which we will denote by 1 as well. ${ }^{1}$ Denote by $\epsilon$ the 'canonical functor' $\mathcal{C} \stackrel{y}{\longrightarrow} \operatorname{Pre}(\mathcal{C}) \stackrel{L}{\longrightarrow}$ $\operatorname{Sh}(\mathcal{C}, J)$, the Yoneda embedding followed by sheafification. Let $\left\{U_{i} \stackrel{u_{i}}{\longrightarrow}\right.$ $\left.\mathbf{1} \mid i \in I_{1}\right\}$ be an arbitrary set of arrows in $\mathcal{C}$ with common target $\mathbf{1}$. Set $\mathcal{U}_{I_{1}}=\bigsqcup_{i \in I_{1}} \epsilon\left(U_{i}\right)$. For another such collection $\left\{U_{k} \stackrel{u_{k}}{\longrightarrow} \mathbf{1} \mid k \in I_{2}\right\}$, a refinement from the latter to the former is a mapping $\Phi: I_{2} \rightarrow I_{1}$ together with a factorization $U_{k} \rightarrow U_{\Phi(k)} \rightarrow \mathbf{1}$ for each $k \in I_{2}$. $\Phi$ then induces a map $\mathfrak{U}_{I_{2}} \rightarrow U_{I_{1}}$. Refinements can be composed in the obvious way.

Note that $\mathcal{U}_{I}=\bigsqcup_{i \in I} \epsilon\left(U_{i}\right) \rightarrow \mathbf{1}$ in $\mathcal{E}$ iff the $U_{i} \stackrel{u_{i}}{\longrightarrow} \mathbf{1}$ form a covering family; in that case, $\operatorname{cosk}_{0}\left(\mathfrak{U}_{I}\right)$ is a 1-cover of $\mathcal{E}$. (Here and hereafter, we write covering family to mean a collection of arrows with common codomain generating a covering sieve for the topology $J$, to ease somewhat on the multiple uses of the term 'cover'.) Let $\operatorname{cech}_{\mathcal{C}, J}^{1}$ be the category whose objects are covering families $\left\{U_{i} \stackrel{u_{i}}{\longrightarrow} \mathbf{1} \mid i \in I\right\}$ and whose morphisms are refinements. (It is a small category if $(\mathcal{C}, J)$ is a small site.) In line with the notation of Proposition 3.12, write $\left[\operatorname{cech}_{\mathcal{C}, J}^{1}\right.$ ] for the category with the same objects as $\operatorname{cech}_{\mathcal{C}, J}^{1}$, but morphisms being (the simplicial maps induced by) refinements, modulo simplicial homotopies.

Recall that a preorder is a reflexive, transitive relation; it can also be thought of as a category whose every hom-set is either empty or a singleton.

PROPOSITION 4.2. $\left[\operatorname{cov}_{\mathcal{E}}^{1}\right]$ is a preorder.

\footnotetext{
${ }^{1}$ For the sake of exposition, this article is concerned only with derived functors of the global section functor, meaning global homotopy classes with source the terminal object; that is the reason for limiting the description to Čech covers of $\mathbf{1}$, though it leads to tautologous notation occasionally.
} 
Proof. Quite generally in $\varepsilon^{\Delta^{\mathrm{op}}}$, any two maps $f, g: X \rightarrow \operatorname{cosk}_{0}(Y)$ into a 0 -coskeletal object are simplicially homotopic: the homotopy $\Delta[1] \times X \stackrel{h}{\longrightarrow} \operatorname{cosk}_{0}(Y)$ comes via adjunction from its 0 -truncation, which is $X_{0} \sqcup X_{0} \stackrel{f_{0} \sqcup g_{0}}{\longrightarrow} Y$.

LEMMA 4.3. Given $\left\{U_{i} \stackrel{u_{i}}{\longrightarrow} \mathbf{1} \mid i \in I_{1}\right\}$, let $\left\{U_{k} \stackrel{u_{k}}{\longrightarrow} \mathbf{1} \mid k \in I_{2}\right\}$ be the sieve it generates. Then $\operatorname{cosk}_{0}\left(\mathcal{U}_{I_{1}}\right)$ and $\operatorname{cosk}_{0}\left(\mathcal{U}_{I_{2}}\right)$ are simplicially homotopy equivalent.

Proof. By Proposition 4.2, it suffices to exhibit a refinement from $I_{2}$ to $I_{1}$, and one in the other direction. Since each element of $I_{2}$ must factor through an element of $I_{1}$ (by definition), a choice of such for each $u_{k} \in I_{2}$ gives a refinement from $I_{2}$ to $I_{1}$. In the other direction one has the inclusion $I_{1} \subseteq I_{2}$.

Let $J(\mathbf{1})$ be the collection of $J$-sieves covering $\mathbf{1}$, ordered by inclusion; it is a cofiltered poset.

PROPOSITION 4.4. J(1) and $\left[\operatorname{cech}_{\mathcal{C}, J}^{1}\right]$ are equivalent as categories.

Proof. By Proposition 4.2, [cech ${ }_{\mathcal{C}, J}^{1}$ ] is a preorder. Since each covering sieve is a covering family, and each inclusion of sieves a refinement, one has a functor $J(\mathbf{1}) \hookrightarrow\left[\operatorname{cech}_{\mathcal{C}, J}^{1}\right]$. It is faithful and full, and surjective on isomorphism types of objects by Lemma 4.3.

Since for any object $T_{0}$ of global support, there exists a covering family $\left\{U_{i} \stackrel{u_{i}}{\longrightarrow} 1\right\}$ that allows a map $\bigsqcup \epsilon\left(U_{i}\right) \rightarrow T_{0}$, and $\left[\operatorname{cov}_{\mathcal{E}}^{1}\right]$ is a preorder, one has

COROLLARY 4.5. The (faithful but not necessarily full) functor $\left[\operatorname{cech}_{\mathcal{C}, J}^{1}\right] \rightarrow$ $\left[\operatorname{cov}_{\mathcal{E}}^{1}\right]$ induced by sending $\left\{U_{i} \stackrel{u_{i}}{\longrightarrow} \mathbf{1}\right\}$ to $\operatorname{cosk}_{0}\left(\bigsqcup \epsilon\left(U_{i}\right)\right)$ is cofinal. For any $X \in \mathcal{E}^{\Delta^{o p}}$, one has a natural bijection

$$
\underset{\left[\operatorname{cech}_{\mathcal{C}, J}^{1}\right]}{\operatorname{colim}}[-, X] \rightarrow \underset{\left[\operatorname{cov}_{\varepsilon}^{1}\right]}{\operatorname{colim}}[-, X]
$$

For $X=K(A, n)$, one can define Čech cohomology of the site $(\mathcal{C}, J)$ with coefficients in $A$ as $\check{H}_{\mathcal{C}, J}^{n}(A)=\underset{\left[\operatorname{cech}_{\mathcal{C}, J}^{1}\right]}{\operatorname{colim}}[-, K(A, n)]$. If iterated products of the objects $U_{i}$ that make up Čech covers exist in $\mathcal{C}$, then the objects of higher-dimensional simplices of the $\operatorname{cosk}_{0}\left(\sqcup \epsilon\left(U_{i}\right)\right)$ are representable, hence $\breve{H}^{n}(A)$ can be computed by the usual recipe, i.e. as a filtered 
colimit of cohomology of chain complexes. The map of Corollary 4.5 followed by $\operatorname{colim}_{\left[\operatorname{cov}_{\varepsilon}^{1}\right]}[-, X] \rightarrow \mathrm{ho}_{\mathcal{E}^{\Delta^{\mathrm{op}}}}(\mathbf{1}, X)$ specializes to the natural transformation $\check{H}^{n}(A) \rightarrow H^{n}(A)$ from Čech to derived functor cohomology, and as a corollary of the main theorem, it is an isomorphism for $n=0,1$.

Remark 4.6. Čech covers can only be defined for a site, and are (covariantly) functorial for site morphisms; here a site morphism from $(\mathcal{D}, K)$ to $(\mathcal{C}, J)$ (let us assume these have finite limits) is a functor $\mathcal{D} \stackrel{F}{\longrightarrow} \mathcal{C}$ that preserves finite limits and takes $K$-covers to $J$-covers. It induces a topos $\operatorname{map} \operatorname{Sh}(\mathcal{C}, J) \rightarrow \operatorname{Sh}(\mathcal{D}, K)$. Recall that any category of sheaves can be presented on a proper class of different sites, and it may happen that $\operatorname{Sh}(\mathcal{C}, J)$ and $\operatorname{Sh}(\mathcal{D}, K)$ are equivalent as categories without there being any site map between $(\mathcal{C}, J)$ and $(\mathcal{D}, K)$. (There will exist a zig-zag of site maps, though.) At any rate, in the absence of Corollary 4.5 it would be far from obvious that the abelian group $\breve{H}_{\mathcal{C}, J}^{n}(A)$ depends purely on the category of sheaves $\operatorname{Sh}(\mathcal{C}, J)$ and the object $A$ in it.

Remark 4.7. Note that Proposition 3.7 was a purely formal consequence of Proposition 3.5; one of the morals of this article is that a choice of (functorial) Postnikov sections, in any sheafifiable homotopy model category, will amount to a theory of truncated hypercovers. The choice of Postnikov functor matters, however. Suppose, for example, that in the proof of Proposition 3.7, one used $\operatorname{cosk}_{n+1} \circ \operatorname{tr}_{n+1}$ in place of Duskin's $\mathcal{P}_{n}$ to truncate both the hypercover $T$ and the target $X$. One will then reach the conclusion that global homotopy classes into the $n+1$-coskeletal object $X$ (which is therefore an $n$-type) can be computed along $n+1$-coskeletal hypercovers. ${ }^{2}$ This (while certainly correct) is not the most economical statement. Specializing to $n=1$, for example, one thus obtains that derived functor $H^{1}$ can be computed along 2-coskeletal hypercovers, while Grothendieck's theorem is that derived functor $H^{1}$ can already be computed along Čech covers, which are 0 -coskeletal.

This 'discrepancy of coskeletal dimension' is due to the curious interaction of exact $n$-types with coskeletal objects (see Proposition 3.4 and Proposition 3.10) and is absent in the formulation in terms of exact $n$-types, as in Theorem 3.15. This is really the only point where a bit of geometry (the geometry of simplices) sneaks into a story that is mainly formal homotopy theory.

Let us move to Čech 2-covers.

${ }^{2}$ This is indeed Proposition 8.9 of Dugger-Hollander-Isaksen [6]. 
EXAMPLE 4.8. Continuing with the setting of Example 4.1,

(0) set $V_{i j}=U_{i} \cap U_{j}$ for each ordered pair $i, j$, and let $\mathcal{V}$ be the coproduct of the $V_{i j}$. The inclusions $V_{i j} \hookrightarrow U_{i}$ resp. $V_{i j} \hookrightarrow U_{j}$ together with $U_{i}=$ $V_{i i}$ define a sheaf of (directed, unit arrow-equipped) graphs $\mathcal{V} \underset{t}{\stackrel{s}{\rightrightarrows}} \mathcal{U}$, the 1 -coskeleton of which is a 2-cover of $X$.

But the graph $\mathcal{V} \underset{t}{\stackrel{s}{\rightrightarrows}} \mathcal{U}$ is nothing but the 1-truncation of the simplicial object $\operatorname{cosk}_{0}(\mathcal{U})$ constructed in Example 4.1(1), and $\operatorname{cosk}_{1}(\mathcal{V} \underset{t}{\stackrel{s}{\rightrightarrows}} \mathfrak{U})$ is canonically isomorphic to $\operatorname{cosk}_{0}(\mathcal{U})$. All this is just an instance of the tautology that an $n$-cover (considered as a simplicial object) is also an $m$-cover for any $m>n$.

(1) Suppose, however, that the family $\mathcal{V}$ is only a refinement of the collection $\left\{U_{i} \cap U_{j} \mid i, j \in I\right\}$ in the following sense: for each ordered pair $i, j \in I$ one has an index set $K_{i j}$, and for each $k \in K_{i j}$ an open subset $V_{k}$ of $U_{i} \cap U_{j}$ such that $\bigcup_{k \in K_{i j}} V_{k}=U_{i} \cap U_{j}$, and each original open $U_{i}$ is included in the collection $K_{i i}$ (this is to ensure that the unit condition can be satisfied). Let $\mathcal{V}$ be the coproduct of the $V_{k}, k \in K_{i j}, i, j \in I$. The inclusions $V_{k} \hookrightarrow U_{i} \cap U_{j} \hookrightarrow U_{i}$ (resp. $U_{j}$ ) give a sheaf of graphs $\mathcal{V} \underset{t}{\stackrel{s}{\rightrightarrows}} \mathcal{U}$ such that $\mathcal{V} \rightarrow \mathfrak{U} \times \mathcal{U}$, and this is our first non-trivial example of a 2-cover. In short, in a 2-cover a second refinement occurs on double intersections.

(2) Let $C \stackrel{p}{\longrightarrow} X$ be a covering space, with $D \stackrel{d}{\longrightarrow} C \times_{X} C$ another covering. $C \times{ }_{X} C$ contains a 'marked' copy of $C$, the diagonal $C \stackrel{\Delta}{\longrightarrow} C \times{ }_{X} C$. Assume $d$ has a section above $\Delta$, so the unit condition is satisfied. The composite $D \stackrel{d}{\longrightarrow} C \times{ }_{X} C \rightrightarrows C$, the double arrows being the projections, is then a 2-cover of $X$. (Even if not a covering space, $D$ is certainly étale and surjective above $C$, and that is all that matters sheaf-theoretically.)

(3) Analogously to Example 4.1, one can have a mixture of (1) and (2), i.e. a different 2-tier covering space system above each open, but any 2 -cover of a space is dominated by one of type (1).

For the case of any site $(\mathcal{C}, J)$ with products, one has

DEFINITION 4.9. A Čech 2-cover (of the terminal object $\mathbf{1}$ of $\mathcal{C}$ ) is a covering family $\left\{U_{i} \stackrel{u_{i}}{\longrightarrow} \mathbf{1} \mid i \in I\right\}$ together with covering families $\left\{V_{k} \stackrel{v_{k}}{\longrightarrow} U_{i} \times U_{j} \mid k \in K_{i j} ; i, j \in I\right\}$ such that $K_{i i}$, for each $i \in I$, contains $U_{i} \times U_{i} \stackrel{\text { id }}{\longrightarrow} U_{i} \times U_{i}$. The 2-cover associated to this data is 
$\operatorname{cosk}_{1}\left(\mathcal{V}_{K} \underset{t}{\stackrel{s}{\rightrightarrows}} \mathcal{U}_{I}\right)$, where $\mathcal{V}_{K}=\bigsqcup_{\substack{k \in K_{i j} \\ i, j \in I}} V_{k}$ and $\mathcal{U}_{I}=\bigsqcup_{i \in I} U_{i} ; s$ has components $V_{k} \stackrel{v_{k}}{\longrightarrow} U_{i} \times U_{j} \stackrel{\mathrm{pr}_{1}}{\rightarrow} U_{i} \rightarrow \mathcal{U}_{I}$, with the other projection for $t$, and a splitting $\mathcal{U}_{I} \rightarrow \mathcal{V}_{K}$ as assumed. A refinement of Čech 2-covers $\mathcal{V}_{K_{2}} \rightrightarrows \mathcal{U}_{I_{2}} \leadsto \mathcal{V}_{K_{1}} \rightrightarrows \mathcal{U}_{I_{1}}$ is given by a function $\Phi: I_{2} \rightarrow I_{1}$ together with factorizations $U_{i} \rightarrow U_{\Phi(i)} \rightarrow \mathbf{1}$ for $i \in I_{2}$, and a family of functions $\Psi_{i j}: K_{i j} \rightarrow K_{\Phi(i) \Phi(j)}$ together with maps $V_{k} \rightarrow V_{\Psi_{i j}(k)}$ such that

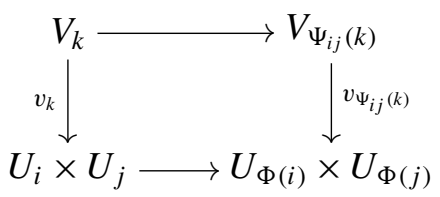

commutes. This will induce a morphism of graphs from $\mathcal{V}_{K_{2}} \underset{t}{\stackrel{s}{\rightrightarrows}} \mathcal{U}_{I_{2}}$ to $\mathcal{V}_{K_{1}} \underset{t}{\stackrel{s}{\rightrightarrows}} U_{I_{1}}$, hence a simplicial map between their 1-coskeleta.

Remark 4.10. If $\mathcal{C}$ does not have products, one can modify the definition appropriately: instead of asking for a covering family $\left\{V_{k} \stackrel{v_{k}}{\longrightarrow} U_{1} \times U_{2} \mid k \in K\right\}$, ask for a set of pairs of maps $\left\{V_{k} \stackrel{v_{k, 1}}{\longrightarrow} U_{1}, V_{k} \stackrel{v_{k, 2}}{\longrightarrow} U_{2} \mid k \in K\right\}$ such that $\bigsqcup_{k \in K} \epsilon\left(V_{k}\right) \stackrel{\epsilon\left(v_{k, 1}\right) \times \epsilon\left(v_{k, 2}\right)}{\longrightarrow} \epsilon\left(U_{1}\right) \times \epsilon\left(U_{2}\right)$ is epi in $\operatorname{Sh}(\mathcal{C}, J)$, etc.

Write $\operatorname{cech}_{\mathcal{C}, J}^{2}$ for the category whose objects are Čech 2-covers, morphisms being refinements, and $\left[\operatorname{cech}_{\mathcal{C}, J}^{2}\right]$ for the category with the same objects, but morphisms the simplicial maps induced by refinement modulo (the equivalence relation generated by) simplicial homotopy. The cheap analogue of Proposition 4.2 fails for $n$-coskeletal objects with $n>0$, and there seems to be no way to construe a canonical poset (or even preorder) of Cech 2-covers on an arbitrary site. The analogue of Corollary 4.5 does survive

PROPOSITION 4.11. [ $\operatorname{cech}_{\mathcal{C}, J}^{2}$ ] is a cofiltered category. The functor $\left[\operatorname{cech}_{\mathcal{C}, J}^{2}\right] \rightarrow\left[\operatorname{cov}_{\mathcal{E}}^{2}\right]$ induced by $\operatorname{cosk}_{1}$ is cofinal.

To prove the first statement, imitate the proof that $\left[\operatorname{cov}_{\varepsilon}^{2}\right]$ is cofiltered using (as Artin and Mazur [2] in fact do) representable sheaves, products of objects of the site and refinements along covering families instead of their intrinsic analogues in the topos $\mathcal{E}$. To prove the second statement, assume given a 2 -cover $T_{1} \rightrightarrows T_{0}$ of $\mathcal{E}$. Find a covering family $\left\{U_{i} \stackrel{u_{i}}{\longrightarrow} 1\right\}, i \in I$, that 
allows a map $\sqcup \epsilon\left(U_{i}\right) \stackrel{u}{\longrightarrow} T_{0}$, and for each pair of indices $i, j \in I$ consider the pullback in $\mathcal{E}$

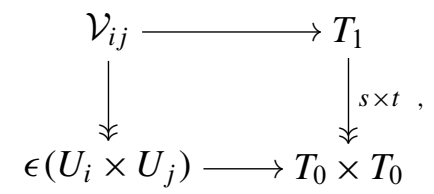

where the bottom horizontal arrow is

$$
\epsilon\left(U_{i} \times U_{j}\right) \hookrightarrow \bigsqcup_{\alpha, \beta \in I} \epsilon\left(U_{\alpha} \times U_{\beta}\right)=\left\{\bigsqcup_{\alpha \in I} \epsilon\left(U_{\alpha}\right)\right\} \times\left\{\bigsqcup_{\beta \in I} \epsilon\left(U_{\beta}\right)\right\} \stackrel{u \times u}{\longrightarrow} T_{0} \times T_{0}
$$

and find a covering family $\left\{V_{k} \stackrel{v_{k}}{\longrightarrow} U_{i} \times U_{j} \mid k \in K_{i j}\right\}$ that refines $\mathcal{V}_{i j}$, i.e. that allows a map $\bigsqcup_{k \in K_{i j}} \epsilon\left(V_{k}\right) \rightarrow \mathcal{V}_{i j}$. Since $\left[\operatorname{cech}_{\mathcal{C}, J}^{2}\right] \rightarrow\left[\operatorname{cov}_{\mathcal{E}}^{2}\right]$ is not necessarily full, and $\left[\operatorname{cov}_{\mathcal{E}}^{2}\right]$ is no longer a preorder, one also needs to verify that given a 2-cover $T_{1} \rightrightarrows T_{0}$ and Čech 2-covers $\left\{U_{i}, V_{k}\right\},\left\{U_{i}^{\prime}, V_{k}^{\prime}\right\}$ that map to it, there exists some Čech 2-cover $\left\{U_{i}^{\prime \prime}, V_{k}^{\prime \prime}\right\}$ refining both $\left\{U_{i}, V_{k}\right\}$ and $\left\{U_{i}^{\prime}, V_{k}^{\prime}\right\}$ that renders

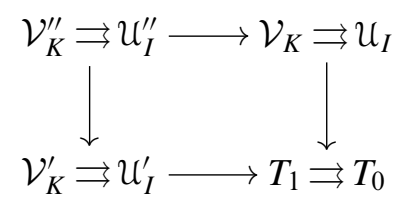

after applying $\operatorname{cosk}_{1}$, commutative in $\left[\operatorname{cov}_{\mathcal{E}}^{2}\right]$. But this is easy, and the above square can in fact be made commutative on the nose: use, first on $T_{0}$ then on $T_{1}$, that the intersection of two covering sieves is a common refinement of each.

The pattern is now quite clear. A Čech 3-cover is the data for a Čech 2-cover $\mathcal{V} \rightrightarrows \mathcal{U}$ together with a refinement of (i.e. epimorphism onto) the object of 2 -simplices of $\operatorname{cosk}_{1}(\mathcal{V} \rightrightarrows \mathcal{U})$. In more detail, let $\mathcal{U}$ be given by the covering family $\left\{U_{i} \stackrel{u_{i}}{\longrightarrow} \mathbf{1} \mid i \in I\right\}$ and $\mathcal{V}$ by the covering families $\left\{V_{\alpha} \stackrel{v_{\alpha}}{\longrightarrow} U_{i} \times U_{j} \mid \alpha \in K_{i j} ; i, j \in I\right\}$. 2-simplices of $\operatorname{cosk}_{1}(\mathcal{V} \rightrightarrows \mathcal{U})$ are triangles in the graph $\mathcal{V} \rightrightarrows \mathcal{U}$, i.e. three edges compatible at the three corners. So consider the set of triples of indices of the form $\left\{\langle\alpha, \beta, \gamma\rangle \mid \alpha \in K_{i j}, \beta \in K_{j k}, \gamma \in K_{i k} ; i, j, k \in I\right\}$. Let $T_{\alpha \beta \gamma}$ be defined as the subobject of $V_{\alpha} \times V_{\beta} \times V_{\gamma}$ that is the intersection of three equalizers; the first of these is the equalizer of $V_{\alpha} \times V_{\beta} \times V_{\gamma} \stackrel{\mathrm{pr}_{1}}{\longrightarrow} V_{\alpha} \rightarrow U_{i} \times U_{j} \stackrel{\mathrm{pr}_{1}}{\longrightarrow} U_{i}$ and $V_{\alpha} \times V_{\beta} \times V_{\gamma} \stackrel{\mathrm{pr}_{3}}{\longrightarrow} V_{\gamma} \rightarrow U_{i} \times U_{k} \stackrel{\mathrm{pr}_{1}}{\longrightarrow} U_{i}$, and similarly for the other two corners. Now give a covering family $\left\{W_{\lambda} \stackrel{w_{\lambda}}{\longrightarrow} T_{\alpha \beta \gamma} \mid \lambda \in L_{\alpha \beta \gamma}\right\}$ for each such 
triple $\langle\alpha, \beta, \gamma\rangle$. (These data are subject to the degeneracy conditions in the case of repeated indices.) One associates a 2-truncated simplicial sheaf to these data, whose 2-coskeleton will be a 3 -cover of $\varepsilon$. The notion of refinement of Čech 3-covers and the properties of the category of Čech 3 -covers and refinements modulo simplicial homotopy can be formulated as expected.

The case of a topological space (or more generally localic topos) is quite visual, since one can restrict attention to subobjects of the terminal object, which form a poset, hence the equalizer conditions become vacuous: a Cech 3-cover is an open cover $U_{i}$, a refinement $\left\{V_{\alpha} \mid \alpha \in K_{i j}\right\}$ of every (nonempty) double intersection $U_{i} \cap U_{j}$, and a refinement of every (non-empty) triple intersection $V_{\alpha} \cap V_{\beta} \cap V_{\gamma}$ with $V_{\alpha} \subseteq U_{i} \cap U_{j}, V_{\beta} \subseteq U_{j} \cap U_{k}, V_{\gamma} \subseteq U_{i} \cap U_{k}$, some $i, j, k$;

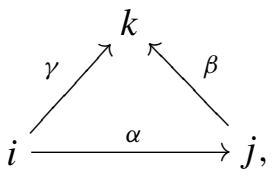

and so on, indexed by boundaries of the standard $n$-simplex ('cocycles').

'Abstract' $n$-covers, as simplicial objects, are more convenient for most proofs, while Čech $n$-covers are better adapted to representing actual data, as in Section 5-though mathematically, of course, the two formalisms are interchangeable.

\section{Examples and Counterexamples}

Let us introduce the notation $\check{H}_{(n)}^{*}$ for level $n$ Čech cohomology; that is, by definition,

$$
\check{H}_{(n)}^{i}(\mathcal{E} ; A)=\underset{\left[\operatorname{cov}_{\varepsilon}^{n}\right]}{\operatorname{colim}}[-, K(A, i)]
$$

for a topos $\mathcal{E}$ (in particular, for sheaves on a topological space). Under this convention, $\check{H}_{(1)}^{*}$ is ordinary Čech cohomology and $\breve{H}_{(\infty)}^{*}$ means (formally) Verdier cohomology. For any $i$, there is a direct system

$$
\check{H}_{(1)}^{i} \rightarrow \check{H}_{(2)}^{i} \rightarrow \check{H}_{(3)}^{i} \rightarrow \cdots \rightarrow \check{H}_{(n)}^{i} \rightarrow \cdots
$$

and the main theorem of this paper says that this system stabilizes for $n \geqslant i$, against $\check{H}_{(\infty)}^{i}=H^{i}$. Let us see by an example that this cannot be improved. To begin with, let us construct a family of topological spaces whose Čech and derived functor cohomology differ. ${ }^{3}$

\footnotetext{
${ }^{3}$ None of the standard texts on algebraic topology or sheaf theory seem to contain such examples, no doubt leaving some students wondering whether the energy involved in proving that they coincide for paracompact Hausdorff spaces is well-spent!
} 
Start with an arbitrary space $X$. Let $X^{+}$be the topological space whose underlying set of points is $X$ together with an extra point, to be denoted ' + ', and whose non-trivial opens are simply the original open sets of $X$. $X^{+}$is contractible via the homotopy $X^{+} \times[0,1] \stackrel{h}{\longrightarrow} X^{+}$defined as

$$
h(x, t)= \begin{cases}x & \text { for } t<1, \\ + & \text { for } t=1 .\end{cases}
$$

If $X$ is compact (as we will assume from now on), then by the proper homotopy invariance of sheaf cohomology, $H^{i}\left(X^{+} ; A\right)=0$ for $i>0$, for all constant coefficients $A$.

Let $S X$ be the space whose underlying set of points is $X$ together with two new points, to be denoted ' + ' and ' - ', and whose non-trivial opens are the following: all the original open sets of $X ; X^{+} \stackrel{\text { def }}{=} X \cup\{+\}$; and $X^{-} \stackrel{\text { def }}{=}$ $X \cup\{-\}$. From the Mayer-Vietoris sequence of the pair of opens $\left\{X^{+}, X^{-}\right\}$, one gets

$$
H^{i+1}(S X ; A)=H^{i}(X ; A)
$$

canonically, for all $i$.

Since the points,+- are contained in unique minimal opens, whose union is all of $S X$, there is an initial(!) open cover of $S X$ under refinement, namely $\left\{X^{+}, X^{-}\right\}$. The Cech cohomology of $S X$ is therefore isomorphic to the cohomology of this single cover: two opens with a non-empty intersection. The nerve of that is a one-simplex and one obtains

$$
\check{H}_{(1)}^{i}(S X ; A)=0
$$

for all $i>0$. If $X$ has non-vanishing $H^{1}, S X$ is the required counterexample with $i=2$, since $H_{(2)}^{2}(S X ; A)=H^{2}(S X ; A)=H^{1}(X ; A)$.

Remark. This example is far from ad hoc. Let $S$ be the 'Sierpiński space', the topological space with two points, one of which is open and the other closed (the closed point acting as basepoint). One has a continuous map $[0,1] \rightarrow S$ that takes $[0,1)$ to the open point and 1 to the closed one. It has been pointed out by Joyal and Moerdijk [16] that $S$ plays the canonical role of 'interval' for a homotopy theory of toposes (canonical to the same extent that 'natural transformation' is the canonical notion of homotopy between functors). Many homotopy-theoretic constructions have a 'toposophic' analogue with the Sierpiński interval replacing $[0,1] ; X^{+}$is the 'Sierpiński cone' on $X$, and $S X$ is the 'Sierpiński suspension' of $X$. (In fact, $X^{+}$is 'Sierpiński contractible', which implies that it has vanishing sheaf cohomology with constant coefficients for all, not just compact, $X$.) 
There is a pretty description of the level 2 cochains representing $H^{2}(S X ; A)$. One has a map from 1-covers of $X$ to 2-covers of $S X$ : it sends the open cover $\mathcal{V}=\left\{V_{\lambda}\right\}$ of $X$ to the open cover $\left\{X^{+}, X^{-}\right\}$of $S X$, with $\left\{V_{\lambda}\right\}$ as refinement of the intersection $X^{+} \cap X^{-}=X$. (Note that 2-covers of this type are cofinal among all 2-covers of $S X$.) This map extends to a homomorphism

$$
\check{H}_{(1)}^{1}(X ; A) \stackrel{\iota}{\longrightarrow} \check{H}_{(2)}^{2}(S X ; A) .
$$

Indeed, a 'level 1 Čech 1-cochain' on $X$ is the same as a map assigning an element of $A$ to each non-empty double intersection in $\mathcal{V}$. A 'level 2 Čech 2-cochain' is the same as the assignment of an element of $A$ to each 2 -simplex of some 2-cover $\operatorname{cosk}_{1}(\mathcal{V} \rightrightarrows \mathcal{U})$. As we saw in Example 4.8(1), such a 2-simplex consists of three elements $U_{1}, U_{2}, U_{3}$ of the open cover $\mathcal{U}$ together with elements $V_{i j}$ of the refined cover of $U_{i} \cap U_{j}$ (here $i, j=1,2,3$, $i<j$ ) such that $V_{12} \cap V_{13} \cap V_{23} \neq \varnothing$.

In the case of the Sierpiński suspension $S X, \mathcal{U}=\left\{X^{+}, X^{-}\right\}$, and (up to symmetry, i.e. interchange of + and - and permutation of indices) all nondegenerate 2-simplices of $\operatorname{cosk}_{1}(\mathcal{V} \rightrightarrows \mathcal{U})$ are of the following type:

$U_{1}=X^{+}, U_{2}=X^{+}, U_{3}=X^{-} ; V_{12}=X^{+}, V_{13}$ is some element of the open cover $\mathcal{V}$ of $X, V_{23}$ is some element of the open cover $\mathcal{V}$ of $X$; such that $V_{13} \cap V_{23} \neq \emptyset$

which is the same data as a non-empty double intersection in $\mathcal{V}$. This being compatible with coboundaries, one can think of the map $\iota$ on the cochain level as simply 'keeping the decorations on double intersections'. Finally, one has a commutative diagram

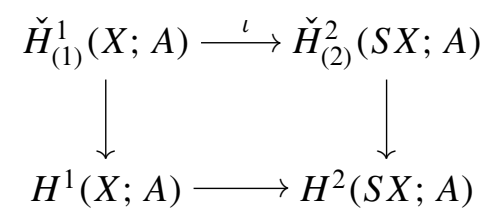

forcing $\iota$ to be an isomorphism, since the other maps are.

One can concoct a great number of related pathologies for spaces with non-closed points. Take, for example, a space containing $n$ opens whose $n$-fold intersection can be refined as $U_{1} \cup U_{2}$ but $U_{1}, U_{2}$ cannot themselves be written as $n$-fold intersections of opens (save tautologously, i.e. by including themselves among the factors): the $n-1$-covers of such a space will not be cofinal among its $n$-covers. Phenomena of this type must be taken into account when defining a supple enough pro-homotopy type for objects $X$ equipped with a Grothendieck topology such that the diagonal $X \stackrel{\Delta}{\longrightarrow} X \times X$ is not closed. In fact, for each $n$ the inverse diagram of 
$n$-covers gives rise to a pro-homotopy type; for $n=1$ one obtains the classical Čech version and for $n=\infty$, the étale homotopy type of Artin and Mazur.

On the 'good' side, for any paracompact Hausdorff space, ordinary open covers are cofinal among $n$-covers for each $n<\infty$ (though not for $n=\infty$ ); this gives an alternative proof of Grothendieck's theorem (see [12]) on the isomorphism of ordinary Čech and derived functor cohomology for such spaces. The argument, being a purely combinatorial one on opens and refinements, extends to other Grothendieck sites. Details will appear in an upcoming paper.

\section{Acknowledgements}

I am indebted to J. Duskin for several email messages and generous simplicial guidance; this work could not have started without his definitions and insights. I also wish to thank the referee for his insistence on making this paper (yet) more practical and concrete.

\section{References}

1. Adámek, J. and Rosický J.: Locally Presentable and Accessible Categories, No. 189, London Math. Soc. Lecture Note Series, Cambridge University Press, Cambridge 1994.

2. Artin, M. and Mazur, B.: Etale Homotopy. No. 100, Lecture Notes in Mathematics, Springer-Verlag, Berlin, 1969.

3. Beke, T.: Simplicial torsors, Theory and Applications of Categories, 9(3) Cambridge, (2001), 43-60.

4. Borceux, F.: Handbook of Categorical Algebra, vol. I-III. Cambridge University Press, Cambridge 1994

5. Brown, K. S.: Abstract homotopy theory and generalized sheaf cohomology, Trans. Am. Math. Soc. 186 (1974), 419-458.

6. Dugger, D. Hollander, S. and Isaksen, D. C.: Hypercovers and Simplicial Presheaves. Math. Proc. Cambr. Phil. Soc., to appear.

7. Duskin, J.: Higher-dimensional torsors and the cohomology of topoi: the abelian theory, In: Applications of Sheaves, No. 753, Lecture Notes in Math., Springer-Verlag, Berlin 1979, pp. 255-279.

8. Duskin, J.: The Azumaya complex of a commutative ring, In: Categorical Algebra and its Applications, No. 1348, Lecture Notes in Math., Springer-Verlag, Berlin 1988, pp. $107-117$.

9. Duskin, J.: Simplicial matrices and the nerves of weak $n$-categories, I. Nerves of bicategories, Theory and Applications of Categories, 9 (2001), 198-308.

10. Gabriel, P. and Zisman, M.: Calculus of Fractions and Homotopy Theory, No. 35, Ergebnisse der Mathematik und ihrer Grenzgebiete, Springer-Verlag, Berlin, 1967.

11. Glenn, P. G. Realization of cohomology classes in arbitrary exact categories, J. Pure and Appl. Algebra, 25(1) (1982), 33-105.

12. Godement, R.: Topologie algébrique et théorie des faisceaux, Hermann, Paris, 1958. 
13. Goerss, P. G. and Jardine, J. F.: Simplicial Homotopy Theory, No. 174, Progress in Mathematics, Birkhäuser Verlag, 1999.

14. Jardine, J. F.: Simplicial objects in a Grothendieck topos, In: Applications of Algebraic K-theory to Algebraic Geometry and Number Theory, Part I, No. 55, Contemporary Mathematics, American Mathematical Society, 1986, pp. 193-239.

15. MacLane, S.: Categories for the Working Mathematician, Springer-Verlag, Berlin, 1971.

16. MacLane, S. and Moerdijk, I.: Sheaves in Geometry and Logic, Springer-Verlag, Berlin, 1992.

17. Makkai, M. and Paré, R.: Accessible categories: the foundations of categorical model theory, No. 104, Contemporary Mathematics, American Mathematical Society, 1989.

18. Quillen, D.: The geometric realization of a Kan fibration is a Serre fibration. Proc. Am. Math. Soc. (19) (1968), 1499-1500. 